\title{
DS-CDMA Systems Performance Using Chaotic Sequence in Different Linear Receivers
}

\author{
Mahdi Sharifi ${ }^{1} \&$ Mohammad Jafar pour jalali ${ }^{2}$ \\ ${ }^{1}$ Dept. of Electrical Engineering, Adiban Institute of Higher Education, Garmsar, Iran \\ ${ }^{2}$ Dept. of Electrical Engineering, AmirKabir University of Technology (Tehran Polytechnic), Tehran, Iran
}

\begin{abstract}
The Direct Sequence-Code Division Multiple Access (DS-CDMA) systems offer physical layer security without the need for a significant increase in computation or power requirements but, these systems suffers from multiple access interference because of other users transmitting in the cell, channel inter symbol interference and additive white Gaussian noise. Codes have an effective role in DS-CDMA system, so M-sequences; gold sequences have been used as spreading codes in DS-CDMA. These sequences by shift registers and periodic in nature are developed. However, these sequences are not enough and also limit the security. This paper presents an investigation on use of new type of sequences called chaotic sequences for DS-CDMA system. These sequences are generated by chaotic maps. First of all, chaotic sequences are easy to generate and store. For very long sequences there are needed only a few parameters and functions. Moreover, numerous numbers of sequences can be developed simply by changing its initial condition. Chaotic sequences are deterministic, reproducible, uncorrelated and random-like, which can be very helpful in enhancing the security of transmission in communication. This paper examines the use of chaotic sequences in DS-CDMA systems using various receiver techniques. Extensive simulation indicate the performance of the different linear DS-CDMA receivers like RAKE receiver, matched filter $(M F)$ receiver and Minimum Mean Square Error (MMSE) receiver using Chaotic Sequences and Gold Sequences.
\end{abstract}

Keywords: Gold sequence, Chaotic Sequence, Direct Sequence-Spread Spectrum (DS-SS), Code Division Multiple Access (CDMA)

\section{INTRODUCTION}

Direct sequence spread spectrum is one of the spread spectrum techniques. Traditionally, a pseudo random (PN) sequence is used for DS-CDMA systems, but it lacks security due to fact that there is limited number of available PN sequences and they show periodic correlation properties. Studies in non-linear dynamical systems have developed chaotic theories. Chaotic sequences, based on chaotic theories are non-binary and non-periodic sequences. The number of available chaotic sequences for DS-CDMA systems can be very large. It is very difficult for an interceptor to decipher the chaotic sequence even if a chaotic function is known. The properties of chaotic sequences provide advantages over the conventional PN sequences based systems. DSCDMA is a multiple access technique based on DS-SS in which multiple users can transmit their data on the same channel using orthogonal spreading sequences. In conventional DS-CDMA, in order to spread the bandwidth of the transmitting signals, PN sequences have been used extensively. It is a deterministic, periodic signal that is known to both transmitter and receiver, whose appearance has the statistical properties of sampled white noise. It appears an unauthorized listener; it is similar to those of white noise [1].

Chaotic behavior is present in many systems where it has been labeled as noise or some internal nonlinear characteristic of the observed system. Chaotic oscillations come from non-linear system elements that cause unwanted behavior. Those chaotic signals have been identified as a component in all man-made and natural complex systems. Research has found that these chaotic oscillations can be reproduced using relatively simple mathematical constructs that led to better understanding of chaotic and non-linear system behavior. Chaotic systems display interesting properties that can be used in digital communication systems. One of these properties is sensitivity to system parameter change and changes to initial conditions. Another property is the random nature of chaotic signals. Both properties can be used to increase the capacity in multiple access systems $[2,3]$. Sensitivity to initial conditions and system parameters can be used to generate sequences from discrete chaotic maps. These sequences have been known to have random characteristics similar to pseudo-random sequences $[2,3$, and 4$]$.

\section{GOLD SEQUENCES}

M-sequences are not optimal for CDMA applications. For CDMA, it is required to construct a family of spreading sequences, one for each which, in which the codes have well-defined cross-correlation properties. In general, m-sequences do not satisfy the criterion. One popular set of sequences is the gold sequences. Gold sequences are attractive because only simple circuitry is needed to generate a large number of unique codes. 


\section{CHAOTIC SYSTEM}

A chaotic dynamical system is an unpredictable, deterministic and uncorrelated system that exhibits noise-like behavior through its sensitive dependence on its initial conditions, which generates sequences similar to PN sequence. The chaotic dynamics have been employed to various engineering applications such as automatic control, signals processing and watermarking. The signals generated from chaotic dynamic systems are noise-like, super sensitive to initial conditions and have spread and flat spectrum in the frequency domain, it is advantageous to carry messages with this kind of signal that is wide band and has high communication security. Then, numerous engineering applications of secure communication with chaos have been developed.

\section{CHAOTIC SEQUENCES}

Chaotic sequence is non-converging and non-periodic sequence that exhibits noise-like behavior through its sensitive dependence on its initial condition. Chaotic systems have sensitive dependence on their initial conditions. A large number of uncorrelated, random-like, yet deterministic and reproducible signals can be generated by changing initial value. These sequences generated by chaotic systems are called chaotic sequences. Chaotic sequences are real valued sequences. Since the spreading sequence in a Chaotic Spread Spectrum (SS) it is no longer binary, the application of the chaotic sequences in DS-CDMA is limited. A further attempt to transform continuous values to binary ones by using digital encoding technique is used to adopt it in DS-CDMA. Some criteria are performed. Moreover, chaotic dynamical system is a deterministic system, disguising modulation as noise would be easily made upon its random-like behavior. The use of chaotic sequences for spectral spreading in a DS-SS has been shown to provide several advantages over conventional binary sequences, particularly pseudo-noise sequences which are used in digital communication.

\section{Generation of ChaOtic Sequence}

One major difference between chaotic sequences and PN are not binary. Therefore chaotic sequences must be transformed into binary sequences. There are various methods of generating binary sequences from chaotic sequences. Different types of binary function are defined to get binary sequences based on a chaoticvalued orbit generated by Ergodic maps.

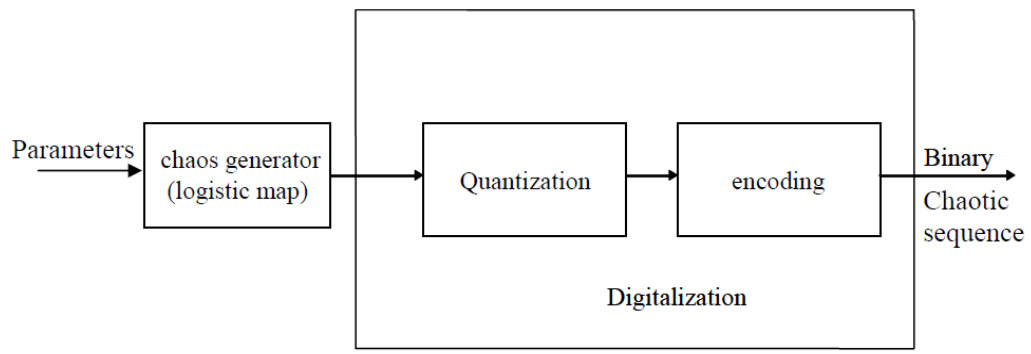

Figure-1. Binary chaotic sequences generator

The block diagram of generation of binary chaotic sequences by this method is given in Figure-1. The chaotic sequences are transmitted into quantization and encoding block. The quantization performs an equalinterval quantization of the floating point input signal varying from -1 to +1 . The output signal is quantized into whole units, the unit size determined by the number of bits used in the binary representation. The coding block converts the quantized signal into a stream of bits. The sequence obtained in this way is called chaotic bit sequence.

\section{MATCHED FILTER (MF)}

MF receiver as simplest receiver is simply the correlator receiver with $\mathrm{M}$ tap weights, matched to the complex conjugate time-reverse of the original spreading sequence of the required user without loss of generality; we may take to be user 1 . The simplest CDMA receiver is the MF receiver, where w is replaced by $\mathrm{Cd}$, spreading sequence vector of the desired user. In practice, the acquisition and synchronization of the chiplevel signal is a highly non-trivial task. A very simple and well known detector for SS signals is the matched filter detector. The matched filter detector basically consists of a tapped-delay-line (TDL) filter of which the number of taps equals the spreading sequence length $\mathrm{N}$. The output vector $(\mathrm{K})$ of the tapped delay line is multiplied with a vector of constant weight. The resulting scalar product is applied to a decision function e.g. a sign function.

For the matched filter, the weights are matched to the user specific sequence code. It provided that the receiver is perfectly synchronized to the transmitter, the TDL extracts a set of chips that represents a particular sequence and the multiplication with the weights is equivalent to dispreading operation. 
A following decision device such as sign function leads to the final estimate of the transmitted data bit, hence. In a single user system, the matched filter is the optimum receiver for signals corrupted by only AWGN. In a multi user environment, however, the performance degrades rapidly with increasing number of users. The matched filter is multiple-access limited-and strong interferers with high power compared to the desired user cause severe problem. This latter effect is called the near-far problem. Due to these problems, other solution has been searched for. The optimal linear receiver for multi-user detection is MMSE receiver and described in the next section.

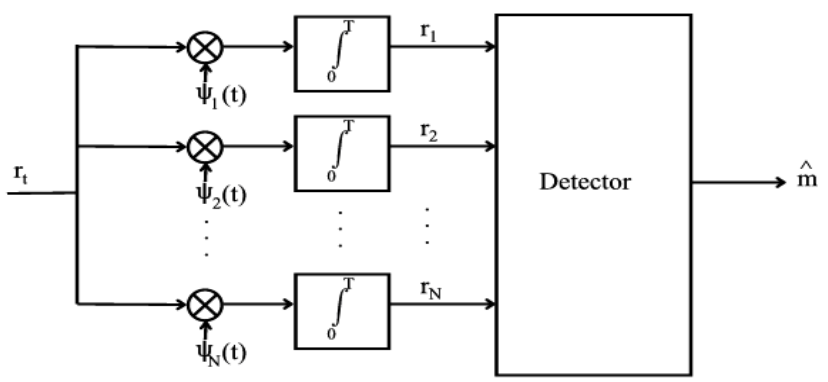

Figure-2. Matched Filter block diagram

\section{MMSE RECEIVER}

The motivation for the use of adaptive algorithms lies in the desire to change the individual taps of the receiver filter to respond to changes in the communication channel. The traditional implementation of adaptive receivers is that a sequence of a priori known training data is incorporated into the data stream at prearranged times. It is important to acknowledge that this effectively reduces the overall data rate of the system, which is the main drawback of this approach. The goal of any adaptive algorithm is to use this training data to force the receiver tap weights to minimize some cost or penalty function, the difference metric between the original data bit and its estimated value. The only requirement for this penalty function is that it be a monotonic increasing function of the absolute value of its argument, with a global minimum at zero. MMSE receiver is an adaptive filter as shown in Figure-3, in which the number of receiver tap weights $\mathrm{Nr}$ is set to length of the spreading code M. The MMSE criteria provide equalizer tap coefficients $\mathrm{w}(\mathrm{k})$ to minimize the mean square error at the equalizer output before the decision device. This condition can be represented as:

$$
\begin{gathered}
\mathrm{J}=\varepsilon|\mathrm{e}(\mathrm{k})|^{2} \\
\mathrm{e}(\mathrm{k})=\mathrm{s}(\mathrm{k}-\mathrm{d})-\mathrm{y}(\mathrm{k})
\end{gathered}
$$

Where e $(\mathrm{k})$ is the error associated with filter output $\mathrm{y}(\mathrm{k})$. However, the MMSE criteria optimize the equalizer weights for minimizing the MMSE under noise and ISI. Minimization of MMSE criteria provides equalizers that satisfy the Wiener criterion. The evaluation the equalizer weights with these criteria requires computation of matrix inversion and the knowledge of the channel, which in most cases is not available. With this penalty function, the resulting target tap weights have been shown to be given by the Wiener filter, so that these algorithms may be viewed as an iterative approximation to the Wiener filter However, adaptive algorithms like LMS and RLS can be used to recursively update the equalizer weights during the training period. Two adaptive methods which employ this least square error penalty function are LMS and RLS algorithms.

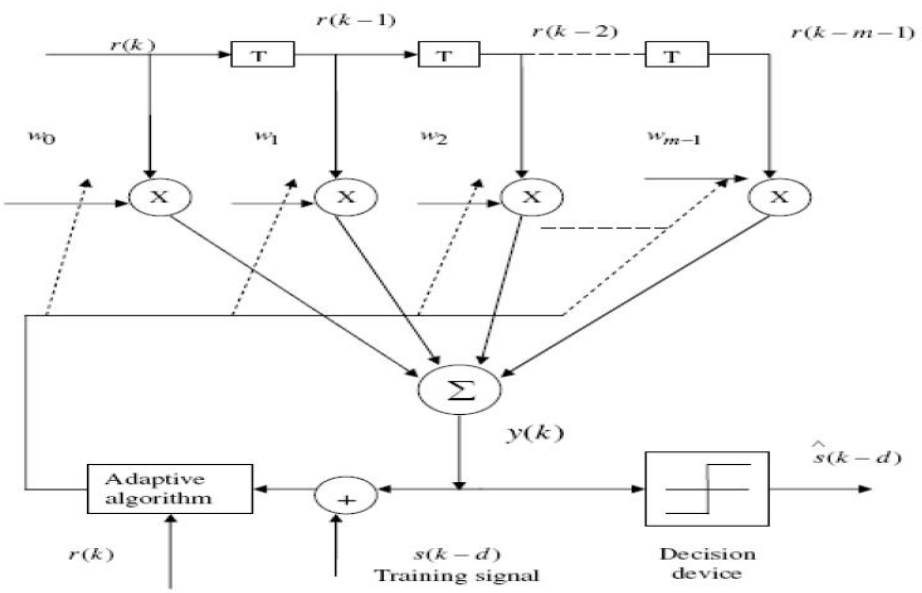

Figure-3. MMSE receiver block diagram 


\section{Simulation RESULTS}

In this section, the BER performance of the different linear receivers like matched filter and MMSE receiver using chaotic spreading sequences is done and the performance is compared with gold sequences. In all the experiments randomly generated +1 and- 1 samples were transmitted for each user. In all the simulations, chaotic spreading sequences and gold sequences of 31 chips are considered. These samples were spread using chaotic spreading sequences of length 31 corresponding to each of the users. For comparison with gold sequences, the maximum permissible user's in the system is restricted to 31. After spreading, the sequences were added and transmitted through the non-dispersive channel. The channel corrupted the transmitted signal with AWGN. The channel output was fed to the various linear receiver structures like Matched filter and MMSE receiver. A total of 105 bits were transmitted by each user and a minimum of 1000 errors were recorded. The tests were conducted for different levels of Eb-N0. Additionally tests were also conducted by varying number of active users in the system for fixed value of Eb-N0.

\section{PERFORMANCE COMPARISON FOR CHANNEL WITHOUT ISI}

Here Eb-N0 was fixed as 7dB. The result shows that chaos based MF receiver performs inferior to gold based MF receiver. It has nearly $3 \mathrm{~dB}$ performance penalty at BER of $10^{-3}$.It is also seen that chaos based MMSE receiver performs inferior to gold based MMSE receiver. It has nearly $1 \mathrm{~dB}$ performance penalty at BER of 10 . The result also shows that chaos based MMSE receiver performs superior to chaos based MF receiver. It has nearly $3 \mathrm{~dB}$ performance penalty at BER of $10^{-3}$. The result also shows that gold based MMSE receiver performs superior to gold based MF receiver. It has nearly $1 \mathrm{~dB}$ performance penalty at BER of $10^{-3}$. It is seen that chaotic sequences performance increases significantly by using MMSE receiver when compared to MF receiver.

In Figure-4 performance of matched filter receiver was investigated for varying Eb-N0 conditions. Performance for chaotic spreading sequences and gold sequences for 4 and 7 users are plotted in Figure-4.10. It is seen that when the number of users is 4 , there is a $2 \mathrm{~dB}$ performance difference at a BER of $10^{-3}$ between chaos based MF and gold based MF receiver. This difference is increased to almost $5 \mathrm{~dB}$ at a BER of $10^{-3}$ in case of 7 users. In both the cases chaotic sequences performance is inferior to gold sequences. For this it is also seen that there is $3 \mathrm{~dB}$ performance penalty at BER of $10^{-3}$ for chaotic sequences based MF and as it seen the number of users increases chaos based MF receiver performance degrades very much when compared to gold based $\mathrm{MF}$ receiver.

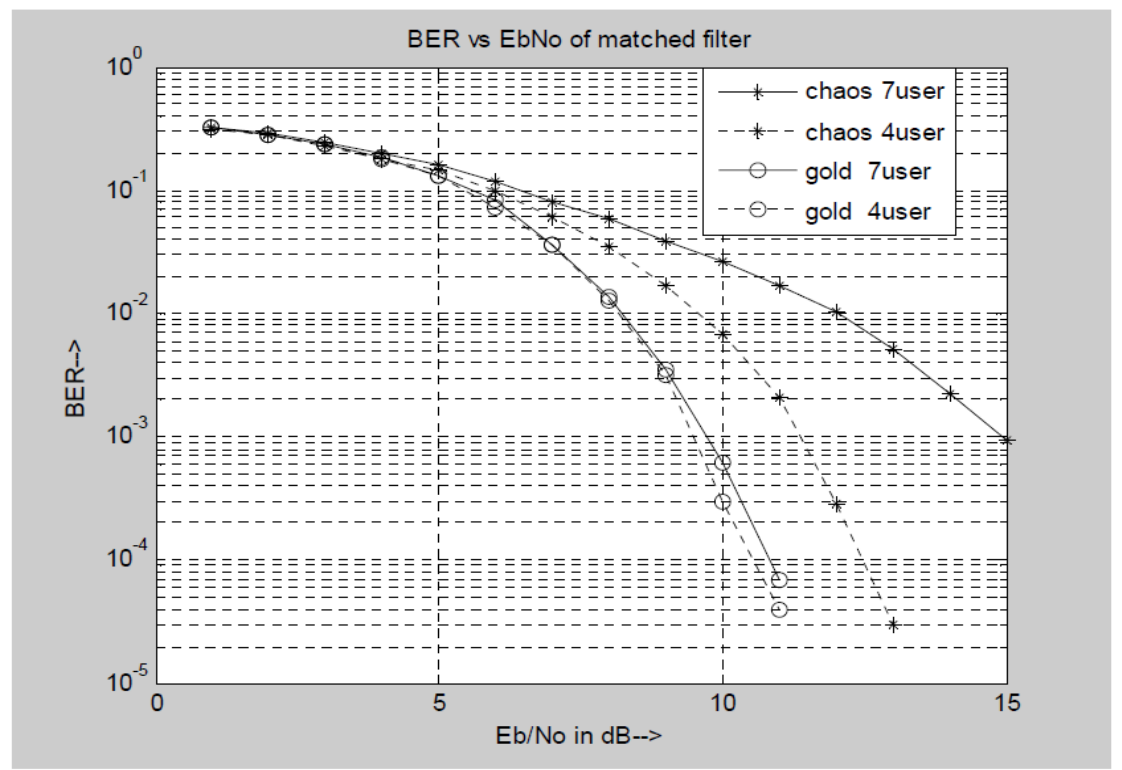

Figure-4. BER performance of Matched Filter 


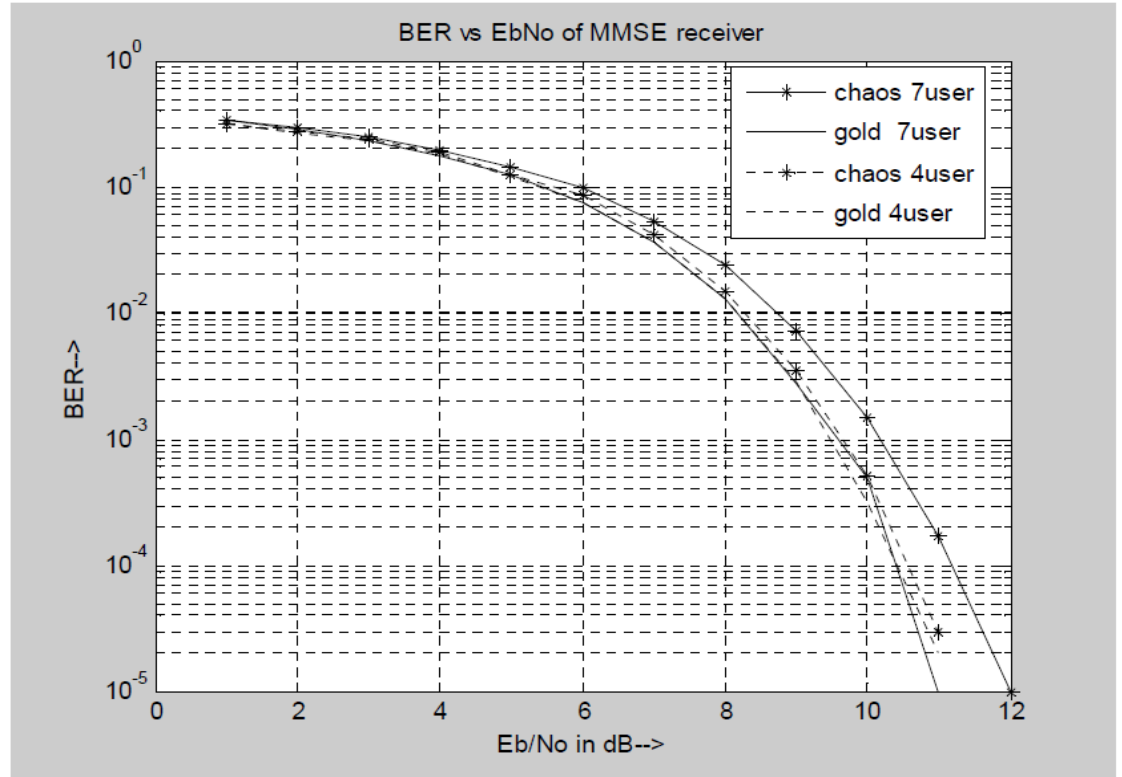

Figure-5. BER performance of MMSE receiver

In Figure-5 performance for chaotic spreading sequences and gold sequences for 4 and 7 users are plotted. It is seen that when the number of users is 4 , there is a $0.2 \mathrm{~dB}$ performance difference at a BER of 10 between chaos based MMSE and gold based MMSE receiver. This difference is increased to almost $1 \mathrm{~dB}$ at a BER of $10^{-3}$ in case of 7 users. In both the cases chaotic sequences performance is very close to gold sequences. For this it is also seen that there is $0.8 \mathrm{~dB}$ performance penalty at BER of $10^{-3}$ for chaotic sequences based MMSE when users are changed from 4 users to 7 users. The number of user's increases chaos based MMSE receiver performance degrades slightly when compared to gold based MMSE receiver.

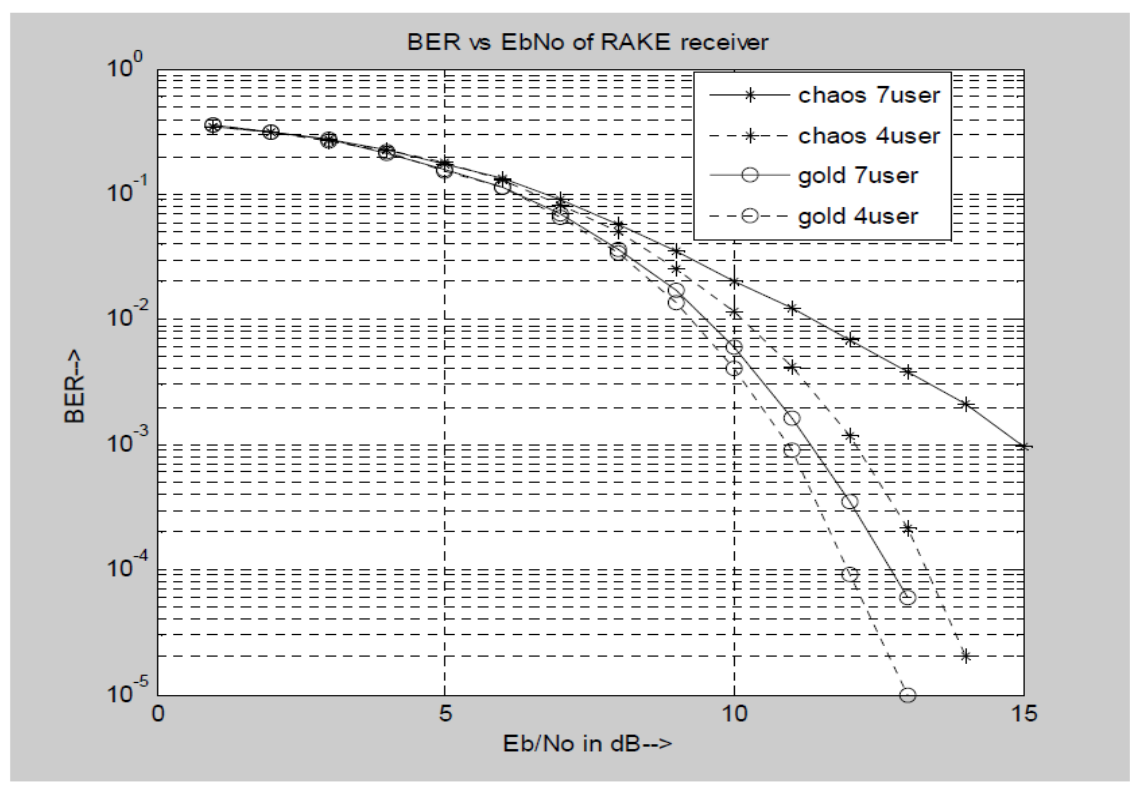

Figure-6. BER performance of RAKE receiver

In Figure-6 performance for chaotic spreading sequences and gold sequences for 4 and 7 users are plotted. It is seen that when the number of users is 4 , there is a $1 \mathrm{~dB}$ performance difference at a BER of 10 between chaos based RAKE and gold based RAKE receiver. This difference is increased to almost $3.5 \mathrm{~dB}$ at a BER of $10^{-3}$ in case of 7 users. 
In both the cases chaotic sequences performance is inferior to gold sequences. For this it is also seen that there is $2.5 \mathrm{~dB}$ performance penalty at BER of $10^{-3}$ for chaotic sequences based. It is also seen that there is $0.5 \mathrm{~dB}$ performance penalty at BER of $10^{-3}$ for gold sequences based RAKE receiver when users are changed from 4 users to 7 users. So as the number of user's increases chaos based RAKE receiver performance degrades very much when compared to gold based RAKE receiver.

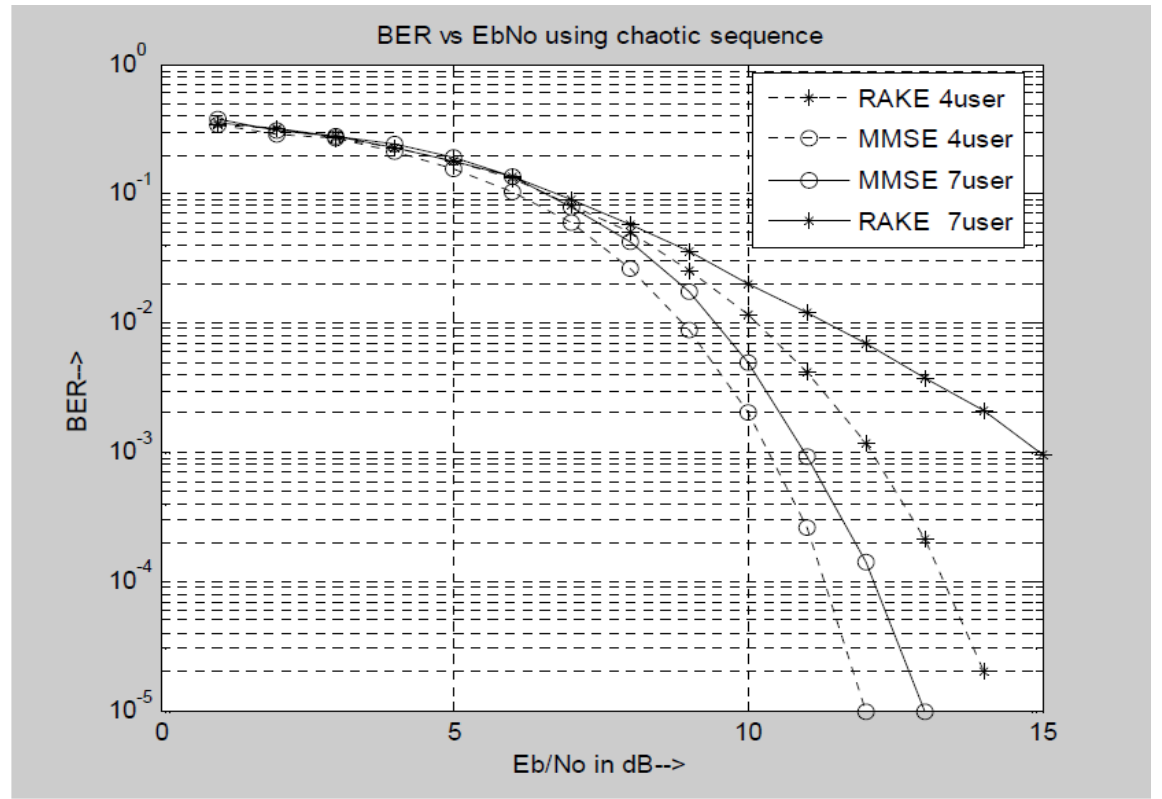

Figure-7. Comparison of BER performance

In Figure-7 performance of different linear receivers was investigated for varying $\mathrm{E}_{\mathrm{b}} / \mathrm{N}_{0}$ conditions. Performance for chaotic spreading sequences for 4 and 7 users are plotted in Figure-8. It is seen that when the number of users is 4 , there is almost $2 \mathrm{~dB}$ performance difference at a BER of $10^{-3}$ between chaos based RAKE and chaos based MMSE receiver. This difference is increased to almost $4 \mathrm{~dB}$ at a BER of $10^{-3}$ in case of 7 users. In both the cases RAKE receiver performance is inferior to MMSE receiver. For this it is also seen that there is $3 \mathrm{~dB}$ performance penalty at BER of $10^{-3}$ for chaotic sequences based RAKE when users are changed from 4 users to 7 users. For this it is also seen that there is only almost $0.7 \mathrm{~dB}$ performance penalty at BER of $10^{-3}$ for chaotic sequences based MMSE when users are changed from 4 users to 7 users. So as the number of user's increases chaos based RAKE receiver performance degrades much when compared to chaos based MMSE receiver. In all the cases it is seen that MMSE receiver performs very well than RAKE receiver.

\section{CONCLUSION}

In this paper various linear receivers like Matched filter, MMSE receiver and RAKE receiver is explained. BER performance of different linear receivers using chaotic sequences is evaluated and it is compared with the receivers using gold sequences. Generation of binary chaotic sequences from different chaotic maps has been discussed. Also, various linear receivers like matched filter, MMSE receiver etc., are studied and BER performance of different linear receivers using chaotic sequences is evaluated and it is compared with the receivers using gold sequences. The results also showed that MMSE receiver performs better than matched filter receiver for chaotic sequence based DS-CDMA.

As a final conclusion it can be stated that although the chaotic sequences based DS-CDMA performance is slight inferior to gold sequences based DS-CDMA, but main advantages of the chaotic sequences in a DS-SS systems such as the availability of a great numbers, the ease of their generation, their inherent improvement in the security of transmission and also the lack of their dependence on the properties of correlation functions can be significantly considered in a DS-CDMA system so that these positive features of the chaotic sequences make strongly itself a viable alternative to PN sequences in terms of generating more effective codes in order to improve the performance and security of the communications based on the DS-CDMA. 


\section{REFERENCE}

[1] R.Gold, "Optimal Binary Sequences for Spread Spectrum Multiplexing,” IEEE. Trans. Inform. Theory.vol. IT-13 , pp.619621.October 1967.

[2] G Heidari-Bateni, CD McGillem, in Wireless Communications, 1992. Conference Proceedings, 1992 IEEE International Conference on Selected Topics in. Chaotic sequences for spread spectrum: an alternative to pn-sequences (IEEEVancouver, 1992), pp. 437440.View ArticleGoogle Scholar

[3] N Rahnama, S Talebi, Performance comparison of chaotic spreading sequences generated by two different classes of chaotic systems in a chaos-based direct sequence code division multiple access system. Communications, IET. 7(10), 1024-1031 (2013).View ArticleGoogle Scholar

[4] VA Kumar, A Mitra, SM Prasanna, on the affectivity of different pseudo-noise and orthogonal sequences for speech encryption from correlation properties. Int. J. Inf. Technol.4 (2), 455-462 (2007).Google Scholar

[5] P. G. Flikkema, "Spread Specrtum Techniques for Wireless Communications," IEEE Signal Processing Magazine, May 1997

[6] Laxmi Bhat and Dr.K.L.Sudha "Performance analysis of chaotic DS-CDMa with CSK Modulation" International Journal of Mobile Network Communications \& Telematics (IJMNCT)

[7] S. Mandal and S. Banerjee, "A chaos-based spread spectrum communication system," Nat. Conf. Nonlinear Sys. Dynamics, Indian Institute of Technology, Kharagpur, Dec 28-30, 2003 